\title{
Part Orientation with One or Two Stable Equilibria Using Programmable Force Fields
}

\author{
Karl Friedrich Böhringer \\ Department of Electrical Engineering \\ University of Washington, Seattle \\ Seattle, WA 98195-2500 \\ karl@ee.washington.edu \\ Lydia E. Kavraki \\ Department of Computer Science \\ Rice University \\ Houston, TX 77005 USA \\ kavraki@cs.rice.edu
}

\author{
Bruce Randall Donald \\ Department of Computer Science \\ Dartmouth College \\ Hanover, NH 03755-3510 \\ brd@cs.dartmouth.edu
}

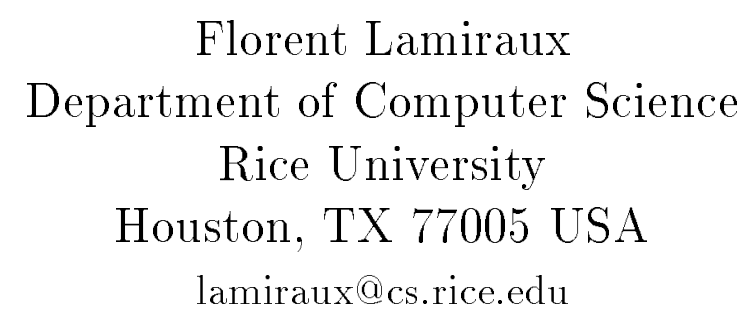 \\ Department of Computer Science Rice University lamiraux@cs.rice.edu}

\begin{abstract}
Programmable force fields are an abstraction to represent a new class of devices for distributed, nonprehensile manipulation for applications in parts feeding, sorting, positioning, and assembly. Unlike robot grippers, conveyor belts, or vibratory bowl feeders, these devices generate force vector fields in which the parts move until they may reach a stable equilibrium pose.

Recent research in the theory of programmable force fields has yielded open-loop strategies to uniquely position, orient, and sort parts. These strategies typically consist of several fields that have to be employed in sequence to achieve a desired final pose. The length of the sequence depends on the complexity of the part.

In this paper, we show that unique part poses can be achieved with just one field. First, we exhibit a single field that positions and orients any part (with the exception of certain symmetric parts) into two stable equilibrium poses. Then we show that for any part there exists a field in which the part reaches a unique stable equilibrium pose (again with the exception of symmetric parts). Besides giving an optimal upper bound for unique parts positioning and orientation, our work gives further evidence that programmable force fields are a powerful tool for parts manipulation.

Our second result also leads to the design of "universal parts feeders", proving an earlier conjecture about their existence. We argue that universal parts feeders are relatively easy to build, and we report on extensive simulation results which indicate that these devices may work very well in practice. We believe that the results in this paper could be the basis for a new generation of efficient, open-loop, parallel parts feeders.
\end{abstract}

\section{Keywords}

programmable force fields, part orientation, MEMS, equilibrium configuration, manufacturing 


\section{INTRODUCTION}

Part manipulation is an important but also time-consuming operation in industrial automation. Parts and, in particular, small parts arrive at manufacturing sites in boxes and they need to be sorted and oriented before assembly. Traditionally part feeding and orienting has been performed with vibratory bowl feeders [48, for example]. These devices are customly designed for the orientation of a single part or a small number of parts and rely on mechanical filters to reject parts in unwanted orientations. Despite their widespread use, vibratory bowl feeders have several disadvantages: they have to be redesigned when the geometry of the part changes; they may damage parts that repeatedly run through the mechanical filters, etc.

Recent work investigates alternative ways for feeding parts in assembly workcells. Parts feeders that are programmed, rather than mechanically modified, offer an attractive solution since they can be used for a wide variety of parts [2, 11, 24, 28]. Practical considerations favor feeding methods that require little or no sensing, employ simple devices, and are as robust as possible $[2,6,11,17,24,25,28,36,50]$. One of the proposed alternatives is the use of programmable force fields $[11,14,33]$. The basic idea is the following: the field is realized on a planar surface on which the part is placed. The forces exerted on the contact surface of the part translate and rotate the part to an equilibrium configuration. The manipulation requires no sensing.

Current technology permits the implementation of certain force fields in the microscale with actuator arrays $[11,14]$ built in micro electro mechanical system (MEMS) technology, and in the macroscale with transversely vibrating plates [5, 6, 11]. The flexibility and dexterity that programmable force fields offer has led researchers to investigate the extent to which these fields can be useful. The work in [11, 14] analyzes the properties of force fields that are suitable for sensorless manipulation and proposes novel manipulation strategies. These strategies typically consist of sequences of force fields that cascade the parts through multiple equilibria until a desired goal state is reached. Figure 1 shows such a two-step sequence to orient a polygonal part (Reprinted from [12]).

Programmable force fields allow us to shift the complexity of parts feeding from the design of mechanical tracks, filters, and cut-outs to control algorithms and circuitry. No sensors or feeder re-design is required. However, the designs proposed in [11, 14] require control software, a clock, and, to some extent, synchronization between distributed actuators. In this paper we show that the device complexity can be further reduced. This work can be seen as an example of minimalist robotics [7, 17], which pursues the following agenda: For a given robot task, find the minimal configuration of resources required 


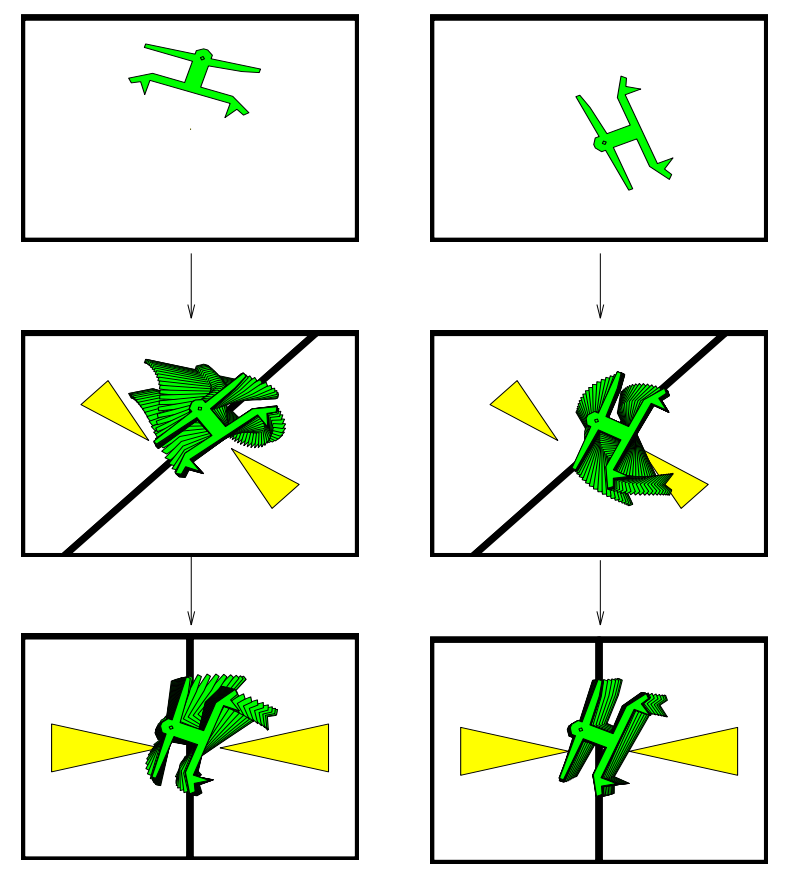

Fig. 1. Sensorless parts orienting using a sequence of force fields: The arrows indicate the direction of the force field. The part reaches unique orientation after applying two subsequent "squeeze fields." There exist such orienting strategies for all polygonal parts. (Reprinted from [12].)

to solve the task. Minimalism is interesting because doing task A without resource B proves that $\mathrm{B}$ is somehow inessential to the information structure of the task. ${ }^{1}$ This paper presents new results on minimalist part feeding, and gives optimal upper bounds on parts positioning and orienting.

Suppose we take the perspective of an architect seeking to simplify a parts feeder. MEMS arrays for programmable force fields require control lines for programmability, plus a clock to switch between control strategies. In addition, control hardware and software are required, for example in a $\mathrm{PC}$ connected to the actuator array. Let us ask the minimalist question: In what ways can be system be simplified? One direction to explore is the following: Does there exist a single field in which every part $P$ has exactly one stable equilibrium (up to part symmetry)? If such a field exists, orientation can be done without

\footnotetext{
${ }^{1}$ In robotics, minimalism has become increasingly influential. Raibert [41] showed that walking and rumning machines could be built without static stability. Erdmann and Mason [26] showed how to do dextrous manipulation without sensing. McGeer [37] built a biped, kneed walker without sensors, computers, or actuators. Canny and Goldberg [17] argue that minimalism has a long tradition in industrial manufacturing, and developed geometric algorithms for orienting parts using simple grippers and accurate, low cost light beams. Brooks [16] has developed online algorithms that rely less extensively on planning and world models. Donald et al. [23, 7] have built distributed teams of mobile robots that cooperate in manipulation without explicit communication. Other related work includes $[19,39,47]$.
} 
sensing and without a clock, achieving minimalism in the corresponding dimensions of resources. It is somewhat remarkable that a purely architectural question can reduce to a conjecture about geometric dynamics.

This paper answers the above questions by presenting two specific device architectures. Assuming non-symmetric parts, the first design achieves exactly two stable equilibria without sensor feedback, clock, or control system. More precisely, unique positioning and orienting is reached modulo $180^{\circ}$ in orientation. The second design overcomes this limitation and for any non-symmetric part achieves unique positioning and orientation. We explain that our second result demonstrates the first known instance of a universal feeder/orienter (UFO) device [11], i.e., a general purpose device that can uniquely position and orient any part without redesigning or reprogramming.

\section{A. Previous Work}

In 1994 [14], Böhringer and Donald proposed that there exist challenging algorithmic problems in MEMS and programmable force fields, at the intersection of combinatorial algorithms, geometry, dynamical systems, and distributed systems. A number of papers (see also Section I-B, below) have emerged on new algorithms, new analysis, and new arrayed devices for programmable force fields. From 1993-1998, Böhringer and Donald worked with Noel MacDonald at the Cornell Nanofabrication Facility to develop and test new arrays of MEMS microactuators for programmable force fields [14, 10, 11, 12]. At the same time, Böhringer and Donald worked with Greg Kovacs' group at the Center for Integrated Systems at Stanford, to develop a control system for MEMS organic ciliary arrays, and to perform experiments with these arrays to manipulate IC dice using array-induced force fields [13, 49]. In parallel, Böhringer and Donald worked with Ken Goldberg at Berkeley and Vivek Bhatt at Cornell to generalize the theory to macroscopic devices, by developing algorithms for transversely vibrating plates in order to implement programmable force fields $[6,5]$. Around this time, Lydia Kavraki explored the power of continuous force vector fields, and demonstrated an elliptical potential field capable of posing any part into one of two equilibrium states [30], and investigated the effect of control uncertainty on the stability of equilibria. Finally, Böhringer and Donald worked with Danny Halperin, to develop new upper and lower bounds, output-sensitive algorithms, and a precise computationalgeometric analysis of the area bisectors arising in squeeze-field algorithms [8]. For other, related papers, see references [5]-[14], [49]. 


\section{B. Related Work}

Until recently, work on force fields for manipulation has been dominated by the artificial potential fields pioneered by Khatib, Koditschek, and Brooks. ${ }^{2}$ While potential fields have been widely used in robot control [31, 32, 42, 46], micro-actuator arrays present us with the ability to explicitly program the applied force at every point in a vector field. ${ }^{3}$ Several groups have described efforts to apply MEMS actuators to positioning, inspection, and assembly tasks with small parts $[3,14,27,33,40$, for example]. However, the fabrication, control, and programming of micro-devices that can interact and actively change their environment remains challenging. ${ }^{4}$

Other groups have also been active in developing new devices, analysis, and algorithms. Ken Goldberg worked with John Canny and Dan Reznik at Berkeley, to continue research on using vibrating plates for manipulation, showing that longitudinal vibrations can generate a rich vocabulary of programmable force fields [45]. In addition, John Canny and Dan Reznik developed sophisticated dynamic models and dynamic simulators for both MEMS devices and macroscopic vibrating plates $[43,44]$. Peter Will and his colleagues at USC-ISI have explored a number of different MEMS array designs, as well as algorithms and analysis for programmable force fields [20, 21, 22, 33]. Andy Berlin, David Biegelsen, and Warren Jackson at Xerox PARC have developed a novel MEMS microactuator array based on controllable air jets, with integrated control and sensing circuitry [4, 18]. Working at CMU, Bill Messner and Jonathan Luntz developed a small room whose floor is tiled with controllable, programmable, macroscopic wheels that can be driven and steered to manipulate large objects such as boxes [34]. Their system employed distributed, local controllers to implement programmable force fields. Together with Howie Choset, they analyzed the resulting dynamical system to obtain interesting results on controllability and programmable force field algorithms based on conservative vs. non-conservative fields [35]. Working with the Berkeley Sensor \& Actuator Center (BSAC), Karl Böhringer and Ken

\footnotetext{
${ }^{2}$ A notable exception are the three-dimensional force fields used by Joffe and his collaborators at JPL [29], where AC magnetic fields are used to orient and assemble ferromagnetic parts - in 3D!

${ }^{3}$ Whereas previous work had developed control strategies with artificial potential fields, our fields are nonartificial (i.e., physical). Artificial potential fields require a tight feedback loop, in which, at each clock tick, the robot senses its state and looks up a control (i.e., a vector) using a state-indexed navigation function (i.e., a vector field). In contrast, physical potential fields employ no sensing, and the motion of the manipulated object evolves open-loop (for example, like a particle in a gravity field). This alone makes our application of potential field theory to micro-devices a different, and algorithmically challenging enterprise.

${ }^{4}$ Problems arise from (1) the limited range of motion and force that can be generated with microactuators, (2) the lack of sufficient sensor information with regard to manipulation tasks, (3) design limitations and geometric tolerances due to the fabrication process, and (4) uncertain material properties and the lack of adequate models for mechanisms at very small scales.
} 
Goldberg explored how MEMS devices employing electrostatic fringing fields can be used to implement programmable force fields for parts manipulation and self-assembly [15].

In short, there has been an explosion of new and exotic arrayed devices for both MEMS manipulation and macroscopic manipulation. The theory of programmable force fields has been applied and extended to a variety of devices and systems. It is somewhat remarkable that the same analysis tools, fields, and algorithms apply to such a wide range of systems. Despite these advances, however, the conjecture [11] about the existence of a Universal Feeder-Orienter (UFO) Device has remained open since 1995; the problem has been widely viewed as resistant to solution. In this paper, we prove the conjecture is true. An earlier version of this proof appeared in [9].

\section{SQueEze Fields and Radial Fields}

In this section we summarize some of the basic results in the theory of programmable force fields that are necessary for the remainder of the paper. In a programmable force vector field, every point in the plane is associated with a force vector in the plane. For example, a unit squeeze field is defined as $\mathbf{f}(x, y)=-\operatorname{sign}(x)(1,0)$. When a part is placed into a squeeze field, it experiences a translation and re-orientation until a predictable equilibrium is reached. This property makes squeeze fields very useful for sensorless positioning and orienting strategies.

Given a polygonal part $P$ with $n$ vertices, it was shown in [14] that there exist $O\left(n^{2} k\right)$ stable equilibrium orientations for $P$ when placed in $\mathbf{f}$ ( $k$ is the number of combinatorially distinct bisector placements for $P^{5}$ ). This result was used to generate strategies for unique parts posing (up to symmetry) by reducing the problem to a parts feeding algorithm developed by Goldberg [28]. The strategies have length $O\left(n^{2} k\right)$ and can be generated in $O\left(n^{4} k^{2}\right)$ time.

In [11] this result was improved to plan lengths of $O(n k)$ and planning time $O\left(n^{2} k^{2}\right)$, by employing combined squeeze and unit radial fields (unit radial fields are defined as $\mathbf{r}(x, y)=\left(-1 / \sqrt{x^{2}+y^{2}}\right)(x, y)$ and are described in more detail in Section $\left.\mathrm{V}\right)$.

The original algorithm in [14] exhibited three key limitations:

1. While unique orientations could be achieved (modulo $180^{\circ}$ ) the final $(x, y)$ position was only known to lie somewhere along the last squeeze axis.

2. The dynamics of the part was assumed to be governed by quasi-static motion with separate phases of translation and rotation ("2PHASE assumption" [14]).

3. Uniqueness of the final orientation was only possible modulo $180^{\circ}$ due to the inherent

\footnotetext{
${ }^{5}$ For details on combinatorially distinct bisector placements see [8].
} 
symmetry in the device design.

The improved algorithm in [11] avoided limitations 1 and 2, but item 3 remained. At the same time the improved algorithms required higher hardware complexity in the device design. In both approaches the part complexity $n$ appears in the upper bounds in the plan complexity, $O\left(n^{2} k\right)$ or $O(n k)$, respectively.

Using elliptic force fields $\mathbf{f}(x, y)=(-\alpha x,-\beta y)$ such that $0<\alpha<\beta$, this bound can be reduced to a constant number (2) independent of $n$. We show this result in Section IV.

It was conjectured in [11] that a field which combines a radial and gravitational field $\mathbf{r}+\delta \mathbf{g}(\mathbf{g}(x, y)=(0,-1)$ and $\delta$ is a small positive constant), has the property of uniquely orienting and positioning parts. We call this field the radial-gravity field and we prove in Section $\mathrm{V}$ that for any non-symmetric part, there is a radial-gravity field inducing exactly one stable equilibrium. Our paper also includes a discussion on implementation issues relating to the radial-gravity field. Such a field could be used to build a universal parts feeder (inspired by the "universal gripper" as proposed by Abell and Erdmann [1] ${ }^{6}$ ). In contrast to the universal manipulator fields proposed in [44], such a device could uniquely position a part without the need of a clock, sensors, or programming.

Table I gives a summary of our results on part manipulation using programmable force fields. The first column of that table specifies a task. The three last columns show the complexity of generating a plan, the number of steps required during plan execution, and the number of final equilibria states for the particular task. The inertial field was defined as $\mathbf{f}(x, y)=-\operatorname{sign}(x)(x, 0)$. In Table I, $n$ denotes the number of vertices of the part and $k$ denotes the combinatorially distinct bisectors of the part.

\section{CONDITIONS FOR EQUILIBRIA}

In this section we give some definitions and establish the notation that will be used in the two following sections. We investigate the conditions for equilibrium for a part $w$ in the presence of a force field $\mathbf{f}: \mathbb{R}^{2} \rightarrow \mathbb{R}^{2}$. It is assumed that $w(x, y) \geq 0$, for $x, y \in \mathbb{R}$, and $W=\int_{\mathbb{R}^{2}} w(\mathbf{p}) d \mathbf{p}<\infty$. Here $w$ can be seen as the support (characteristic) function of the part, this function is 1 on the part and 0 elsewhere. We assume that the support of $w$ is compact.

Without loss of generality, the origin of the reference frame in the plane can be chosen as the center of mass of $w$ :

$$
\int_{\mathbb{R}^{2}} \mathbf{p} w(\mathbf{p}) d \mathbf{p}=\mathbf{0}
$$

\footnotetext{
${ }^{6}$ In a universal gripper a part is free to rotate after being picked up from an arbitrary initial state. Its center of mass will settle at the unique minimum of potential energy, causing the part to reach a unique, predictable equilibrium.
} 


\begin{tabular}{|c|c|c|c|c|c|}
\hline \multirow[t]{2}{*}{ Task } & \multicolumn{2}{|r|}{ Field(s) } & \multicolumn{3}{|c|}{ Complexity } \\
\hline & description & properties & planning & $\begin{array}{l}\text { plan } \\
\text { length }\end{array}$ & $\begin{array}{l}\text { \# of goal } \\
\text { equilibria }\end{array}$ \\
\hline translate & constant & $\begin{array}{l}\text { constant magnitude and di- } \\
\text { rection }\end{array}$ & $\overline{0}$ & 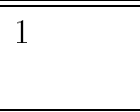 & $\overline{0}$ \\
\hline position & $\begin{array}{l}\text { radial [11] } \\
\text { nad }\end{array}$ & $\begin{array}{l}\text { constant magnitude, continu- } \\
\text { ous directions }\end{array}$ & 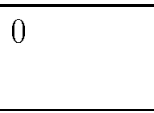 & $\overline{11}$ & $11^{(a)}$ \\
\hline \multirow[t]{2}{*}{ orient } & $\begin{array}{ll}\text { sequence } & \text { of } \\
\text { squeezes }[14] & \\
\end{array}$ & $\begin{array}{l}\text { piecewise constant magnitude } \\
\text { and direction }\end{array}$ & $O\left(k^{2} n^{4}\right)$ & $\overline{O\left(k n^{2}\right)}$ & $2^{(b)}$ \\
\hline & inertial [30] & $\begin{array}{l}\text { smooth magnitude, piecewise } \\
\text { constant direction }\end{array}$ & $O(1)^{(c)}$ & 1 & $2^{(b)}$ \\
\hline \multirow[t]{4}{*}{$\begin{array}{l}\text { position } \\
\text { and } \\
\text { orient }\end{array}$} & $\begin{array}{l}\text { sequence of orthog- } \\
\text { onal squeeze pairs } \\
{[5]}\end{array}$ & $\begin{array}{l}\text { piecewise constant magnitude } \\
\text { and direction }\end{array}$ & $\bar{O} O\left(k^{2} n^{4}\right)$ & $\overline{O\left(k n^{2}\right)}$ & $22^{(d)}$ \\
\hline & $\begin{array}{l}\text { sequence of radial }+ \\
\text { squeeze }[11]\end{array}$ & $\begin{array}{l}\text { piecewise continuous magni- } \\
\text { tude and direction }\end{array}$ & $O\left(k^{2} n^{2}\right)$ & $O(k n)$ & $2^{(d)}$ \\
\hline & elliptic & $\begin{array}{l}\text { smooth magnitude and direc- } \\
\text { tion }\end{array}$ & $O(1)^{(c)}$ & 1 & $2^{(d)}$ \\
\hline & radial-gravity & $\begin{array}{l}\text { smooth magnitude and direc- } \\
\text { tion }\end{array}$ & $O(1)^{(e)}$ & 1 & 1 \\
\hline
\end{tabular}

TABLE I. Fields and algorithms for manipulation tasks with programmable force fields. The results on elliptic and radial-gravity fields are proven in this paper. Remarks:

(a) Translation equilibrium only, orientation is unconstrained.

(b) Orientation unique modulo $180^{\circ}$ symmetry, translation along squeeze line is unconstrained.

(c) Requires numerical computation of axes of inertia.

(d) Pose is unique modulo $180^{\circ}$ symmetry.

(e) Requires numerical computation of field parameter $\delta$.

When the part is in configuration $q=(x, y, \theta)$, the resultant force is given by

$$
\mathbf{F}=\int_{\mathbb{R}^{2}} w(\mathbf{p}) \mathbf{f}\left(A_{\theta} \mathbf{p}+\mathbf{t}\right) d \mathbf{p}
$$

and the resultant torque at the center of mass is given by

$$
\mathbf{M}=\int_{\mathbb{R}^{2}} w(\mathbf{p})\left(A_{\theta} \mathbf{p}\right) \times \mathbf{f}\left(A_{\theta} \mathbf{p}+\mathbf{t}\right) d \mathbf{p}
$$

where $\mathbf{t}=(x, y)^{\top}$, and

$$
A_{\theta}=\left(\begin{array}{ll}
\cos \theta & -\sin \theta \\
\sin \theta & \cos \theta
\end{array}\right)
$$

is the rotation matrix of angle $\theta$. From now on, all integrals extend over $\mathbb{R}^{2}$ unless otherwise stated. 
A total equilibrium is achieved when the resultant force and torque on the part is zero. For a total equilibrium the following two equations must hold:

$$
\begin{aligned}
\mathbf{F} & =\mathbf{0} \\
\mathbf{M} & =\mathbf{0} .
\end{aligned}
$$

\section{Two Stable Equilibrium Orientations}

In this section we show a force field that can orient most parts into two stable equilibria. The field derives from an elliptic potential field and we will call it the elliptic field:

$$
\mathbf{f}(x, y)=(-\alpha x,-\beta y)
$$

where $\alpha$ and $\beta$ are two distinct positive constants. Without loss of generality let us assume that $\alpha<\beta$. Figure 2 displays one such force field with $\alpha=1$ and $\beta=2$. Note that this vector field is the negative gradient of the elliptic potential function $u(x, y)=\frac{\alpha}{2} x^{2}+\frac{\beta}{2} y^{2}$. This potential function is plotted in Figure 3 , for $\alpha=1$ and $\beta=2$.

\section{A. Force and Moment Equilibrium}

\section{A.1 Force Equilibrium}

We first establish the condition for the force equilibrium. If $(x, y)$ are the coordinates of the center of mass of $w$ in configuration $\mathbf{q}$, ( $\mathrm{W}$ is defined in Section III), the total force exerted on $w$, given by the left hand side of (1), is equal to

$$
(-\alpha W x,-\beta W y)
$$

Condition ( 1$)$ is thus equivalent to $(x, y)=(0,0)$. Therefore, in looking for equilibrium configurations $\mathbf{q}$, we only need to consider the configurations of the type $\mathbf{q}=(0,0, \theta)$.

\section{A.2 Moment Equilibrium}

We now proceed to the investigation of condition (2). It turns out that, for "most" parts $w$ and for whatever distinct positive values of $\alpha$ and $\beta$ with $\alpha<\beta$, there are exactly 4 values of $\theta$ for which (2) holds.

Taking into account the force equilibrium, the expression of the torque becomes now

$$
\mathbf{M}=\int w(\mathbf{p})\left(A_{\theta} \mathbf{p}\right) \times \mathbf{f}\left(A_{\theta} \mathbf{p}\right) d \mathbf{p}
$$




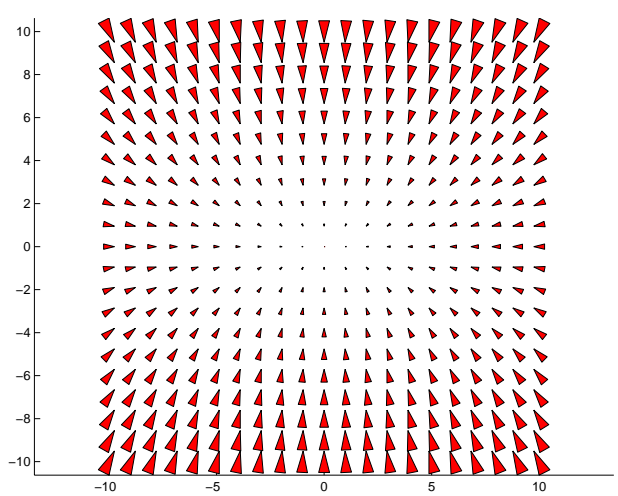

Fig. 2. Force field for $\alpha=1$ and $\beta=2$.

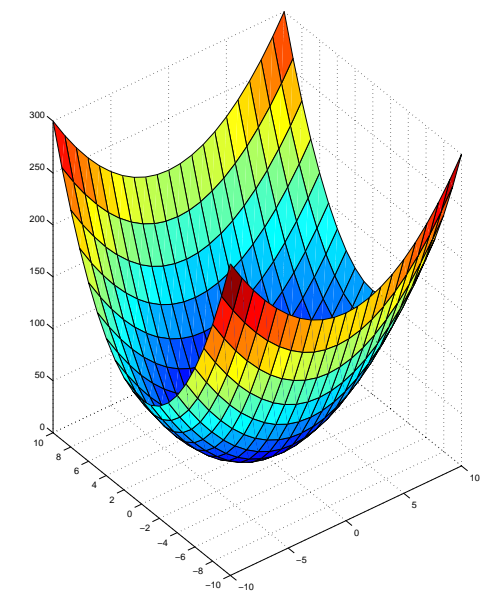

Fig. 3. Elliptic potential function for $\alpha=1$ and $\beta=2$.

The cross product of two vectors $\mathbf{v}_{1}=\left(x_{1}, y_{1}\right)$ and $\mathbf{v}_{2}=\left(x_{2}, y_{2}\right)$ is defined as $\mathbf{v}_{1} \times \mathbf{v}_{2}=$ $\left|\begin{array}{ccc}\mathbf{i} & \mathbf{j} & \mathbf{k} \\ x_{1} & y_{1} & 0 \\ x_{2} & y_{2} & 0\end{array}\right|$ and the above equation gives after calculations

$$
\begin{aligned}
\mathbf{M}= & (\alpha-\beta)\left(\frac{\sin 2 \theta}{2} \int\left(x^{2}-y^{2}\right) w(x, y) d x d y\right) \cdot \mathbf{k}+ \\
& (\alpha-\beta)\left(\cos 2 \theta \int x y w(x, y) d x d y\right) \cdot \mathbf{k} .
\end{aligned}
$$

Thus, since $\alpha \neq \beta$, we have $\mathbf{M}=\mathbf{0}$ if and only if

$$
\frac{s_{20}-s_{02}}{2} \sin 2 \theta+s_{11} \cos 2 \theta=0 .
$$

In the above

$$
s_{m n}=s_{m n}(w)=\int_{\mathbb{R}^{2}} x^{m} y^{n} w(x, y) d x d y
$$

define moments of $w$ and for any real part these quantities are finite. 
Equivalently, we want the vectors

$$
(\cos 2 \theta, \sin 2 \theta) \text { and }\left(s_{11}, \frac{1}{2}\left(s_{20}-s_{02}\right)\right)
$$

to be orthogonal. We now have to distinguish two cases.

"SYMMETRY": $s_{11}=0$ and $s_{02}=s_{20}$.

Clearly in this case (5) is satisfied for all $\theta \in[0,2 \pi)$ and we have equilibrium regardless of orientation. When a part is in equilibrium for all $\theta$, we say that orientation fails for the part.

"ASYMMETRY": $s_{11} \neq 0$ or $s_{02} \neq s_{20}$.

When $\theta$ goes from 0 to $2 \pi$ the vector $(\cos 2 \theta, \sin 2 \theta)$ traverses the unit circle twice. The two vectors, $(\cos 2 \theta, \sin 2 \theta)$ and $\left(s_{11}, \frac{1}{2}\left(s_{20}-s_{02}\right)\right)$ will be orthogonal for exactly 4 values of $\theta$, say $\theta_{1}=\theta_{0}, \theta_{2}=\theta_{0}+\pi, \theta_{3}=\theta_{0}+\frac{\pi}{2}$, and $\theta_{4}=\theta_{0}+\frac{3 \pi}{2}$. In addition, either the first pair of them is stable and the second unstable, or vice versa. The reason is that the sign of $\mathbf{M}$ in (4) determines the direction in which moment $\mathbf{M}$ rotates the part. If this sign is positive, $\mathbf{M}$ rotates the part counter-clockwise, else the rotation is done clockwise (see also [11]). While $(\cos 2 \theta, \sin 2 \theta)$ is rotated around the vector $\left(s_{11}, \frac{1}{2}\left(s_{20}-s_{02}\right)\right)$, the sign of the left hand side of (5) changes after the two vectors attain an orthogonal orientation. Hence, we observe sign changes of the left hand side of (5) for the 4 values of $\theta$ given above. Let $\theta_{1}$ and $\theta_{2}$ be the roots of (5) for which the sign of its left hand side changes from a negative value to a positive value while moving in a counter-clockwise direction. Since we assumed that $\alpha-\beta<0, \theta_{1}$ and $\theta_{2}$ indicate stable equilibrium configurations of the part (see equation 4 ), whereas $\theta_{3}$ and $\theta_{4}$ are unstable configurations.

This leads to the following theorem.

Theorem 1 Let $w: \mathbb{R}^{2} \rightarrow \mathbb{R}$ be a part with finite $s_{i j}$ with $i+j \leq 2$ and whose "center of mass" is at $\mathbf{0}$, and let $\mathbf{f}(x, y)=(-\alpha x,-\beta y)$, with $0<\alpha<\beta$, be the underlying force field.

"SYMmetry": If $s_{11}=s_{20}-s_{02}=0$ the part $w\left(A_{\theta} \mathbf{p}+\mathbf{t}\right)$ is at (force and moment) equilibrium whenever $\mathbf{t}=\mathbf{0}$.

"ASYMMETRY": Otherwise, the distribution $w\left(A_{\theta} \mathbf{p}+\mathbf{t}\right)$ is in equilibrium only when $\mathbf{t}=0$ and for exactly 4 distinct values of $\theta \in[0,2 \pi)$. These 4 values of $\theta$ are $\frac{\pi}{2}$ apart and only 2 of them, say $\theta_{0}$ and $\theta_{0}+\pi$, represent stable equilibria, the others, $\theta_{0}+\frac{\pi}{2}$ and $\theta_{0}+\frac{3 \pi}{2}$ being unstable.

\section{B. Prediction of Equilibria}

In practice, we seek to orient a finite part and it is very easy to compute with numerical techniques the values of $s_{11}, s_{20}$, and $s_{02}$. We can thus predict, for a given part, whether it 


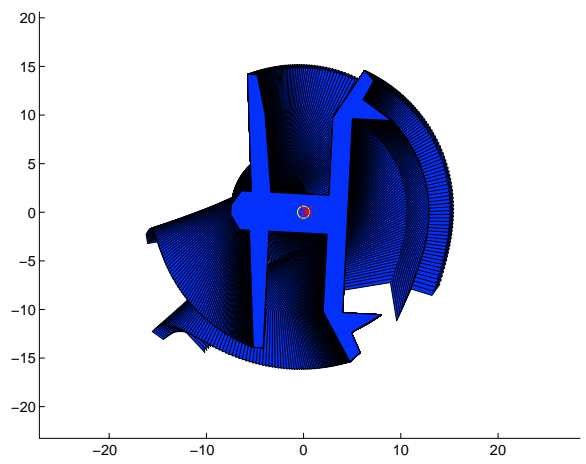

Fig. 4. Orientation of a polygonal part under the elliptic force field the $\alpha=1$ and $\beta=2$.

will have 2 stable equilibria in the force field considered. The equilibrium orientations can be calculated using (5). Note that the equilibrium configurations of a part are independent of $\alpha$ and $\beta$, as long as $0<\alpha<\beta$.

Figure 4 shows the orientation of a polygonal part, called the ratchet, under the elliptic field with $\alpha=1$ and $\beta=2$.

In many cases it is clear that a part will have many equilibrium orientations. For example, consider a planar part that is a regular $n$-gon. This part will be at equilibrium when its "center of mass", as defined in Section III, is at $\mathbf{0}$ no matter what its orientation is. The "center of mass" in this case is the center of its $n$-gon surface. Suppose now that the part had only two equilibria $\theta_{0}$ and $\theta_{0}+\pi$ and that the part is at equilibrium $\theta_{0}$. If we rotate the part by $\frac{2 \pi}{n}$ then we should have an equilibrium again, due to the geometrical symmetry of the part. Hence, since this part can not have only two equilibrium orientations it must be in equilibrium for any value of $\theta$, according to Theorem 1 . Indeed, for this part, it can be shown that $s_{11}=s_{20}-s_{02}=0$. Note that symmetry and asymmetry as in the above theorem do not always correspond to the notion of geometric symmetry and asymmetry, i.e. there may exist parts that are not geometrically symmetric but are symmetric according to the definitions above.

\section{B.1 Equilibria, Principal Axes, and Symmetry}

The constructive proof of Theorem 1 provides a method to predict the stable and unstable equilibria of any two-dimensional part $w$. For a given $w$ we determine its center of mass $\mathbf{c}$ and the angles $\theta_{1}, \ldots, \theta_{4} . w$ is in stable equilibrium in the force field $\mathbf{f}$ if and only if the line through $\mathbf{c}$ at angle $\theta_{1}$ coincides with the $x$-axis.

Readers familiar with theoretical mechanics will recognize the analogy between the proof of Theorem 1 and the transformation equations for moments and products of inertia. These 
equations are the basis for the argument that the principle axes of any two-dimensional part are perpendicular. It is worthwile to explore this analogy in more detail. For any part, there exists a coordinate frame such that $s_{11}=0$. The axes of this coordinate frame are the principal axes of inertia of the part (i.e., axes with maximum or minimum moment of inertia). It can be shown that these axes intersect at the center of mass $\mathbf{c}$. From the previous computations, it is easy to deduce that in the two stable configurations, these axes are lined up with the axis of the force field. More specifically, $s_{20}$ and $s_{02}$ are the second area moments of $w$, often denoted $I_{x}$ and $I_{y}$, and $s_{11}=I_{x y}$ is the product of inertia. The line through $\mathbf{c}$ at angles $\theta_{1}$ or $\theta_{2}$ (corresponding to the stable equilibrium) is the major principal axis, and the line through $\mathrm{c}$ at angles $\theta_{3}$ or $\theta_{4}$ (corresponding to the unstable equilibrium) is the minor principal axis. These observations explain why the equilibrium is independent of the values of $\alpha$ and $\beta$ as long as $0<\alpha<\beta$.

Since all axes of symmetry are principal axes, it further follows that a sufficient condition for "SYMMETRY" as defined in Theorem 1 is that $w$ has two non-perpendicular axes of symmetry. Conversely, a necessary condition for "SYMmETRY" is that the product of inertia of $w$ must be zero for any axis through $\mathbf{c}$, and that the moment of inertia is equal for all axes through $\mathbf{c}$. For more details on principal axes and moments of inertia, see for example [38].

\section{One Stable Equilibrium Orientation}

We now exhibit a class of force fields that induce one stable equilibrium for most parts. These fields are combinations of a unit radial and gravity field and we will call them radial-gravity fields:

- A unit radial field $R$ is defined by: $\mathbf{r}(x, y)=-\frac{1}{\sqrt{x^{2}+y^{2}}}(x, y)$.

- A unit gravity field $\mathbf{g}$ is given by $\mathbf{g}(x, y)=(0,-1)$.

- For a given $\delta \in \mathbb{R}$, the radial-gravity field is defined as the sum of a unit radial field $\mathbf{r}$ and a gravity field $\mathrm{g}$ scaled by $\delta: F_{\delta}=\mathbf{r}+\delta \mathbf{g}$.

Figures 5 and 6 plot a radial-gravity field for which $\delta=0.4$.

\section{A. Force and Moment Equilibrium}

In this section we reason with potential fields instead of using directly equations (1) and (2). First we notice that $F_{\delta}$ derives from the potential field $u_{\delta}(x, y)=\sqrt{x^{2}+y^{2}}-\delta y$ and we define the following potential field over the configuration space $\mathcal{C}$ of the part:

$$
U_{\delta}(q)=\int w(\mathbf{p}) u_{\delta}\left(A_{\theta} \mathbf{p}+\mathbf{t}\right) d \mathbf{p}
$$




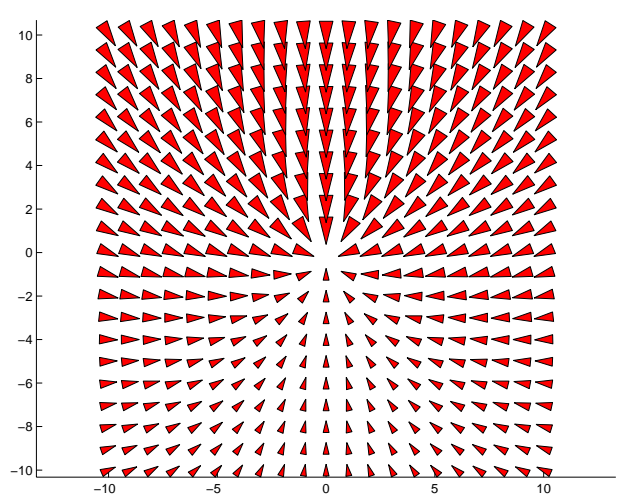

Fig. 5. Radial-gravity field with $\delta=0.4$.

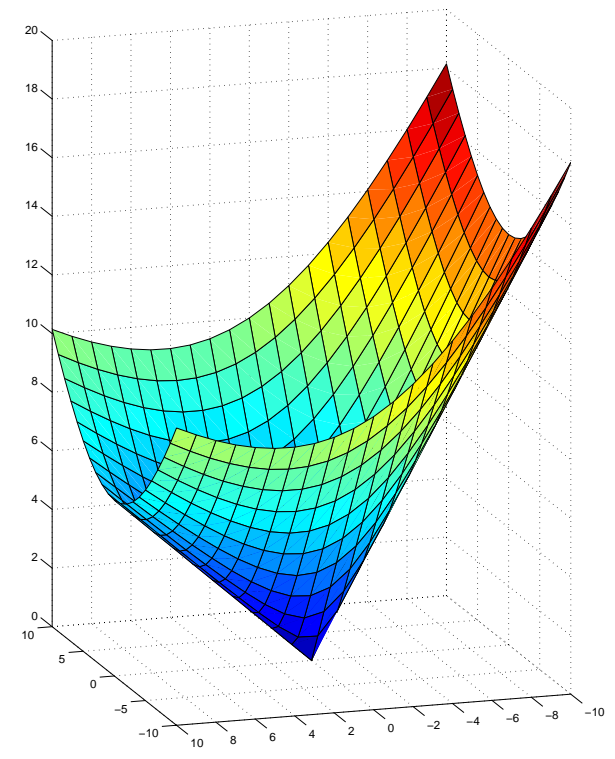

Fig. 6. Combination of a unit radial and a gravitational potential field with $\delta=0.4$.

A configuration $q$ is a stable equilibrium of the part if and only if $q$ is a local minimum of the function $U_{\delta}$.

In order to take advantage of the radial symmetry of $\mathbf{r}(x, y)$, we define a new system of coordinates $(X, Y, \theta)$ from the standard one by

$$
\begin{aligned}
& X=x \cos \theta+y \sin \theta \\
& Y=-x \sin \theta+y \cos \theta .
\end{aligned}
$$

The expression of $U_{\delta}$ in this new system of coordinates is obtained by a change of variable in the integral:

$$
U_{\delta}(X, Y, \theta)=\int w(\xi, \eta) \sqrt{(X+\xi)^{2}+(Y+\eta)^{2}} d \xi d \eta-\delta W(X \sin \theta+Y \cos \theta) .
$$


To establish the existence and uniqueness of a stable equilibrium, we proceed in two steps. First we state the existence and uniqueness of a local minimum of the potential field for any fixed $\theta$. This partial minimum is the force equilibrium. Then we study the curve of force equilibria when $\theta$ describes $\mathbf{S}^{1}$ and reason about moment equilibria. For our discussion below, we define the following functions:

$$
U_{\theta, \delta}(X, Y)=U(X, Y, \theta, \delta)=U_{\delta}(X, Y, \theta)
$$

\section{A.1 Force Equilibrium}

A force equilibrium is a local minimum of $U_{\theta, \delta}$. Using common results of the theory of integration, we find that $U$ is of the class $C^{2}$ and that its partial derivatives with respect to $X$ and $Y$ are obtained by differentiating under the integral. The following proposition establishes the existence and uniqueness of a stable force equilibrium for a fixed $\delta$ by proving that the function $U_{\theta, \delta}$ is convex.

Proposition 2 If $\delta<1, U_{\theta, \delta}$ has a unique local minimum.

Proof: We first notice that for $\delta<1, U_{\theta, \delta}$ tends toward infinity with $(X, Y)$. We show then that $U_{\theta, \delta}$ is convex, i.e. the Hessian of $U_{\theta, \delta}$ is positive definite, that is its eigenvalues are both positive. This condition is fulfilled iff the trace and determinant of the Hessian are both positive:

$$
\begin{aligned}
\operatorname{det} \operatorname{Hess} U_{\theta, \delta}(X, Y) & =\left|\begin{array}{ll}
\frac{\partial^{2} U_{\theta, \delta}}{\partial X^{2}}(X, Y) & \frac{\partial^{2} U_{\theta, \delta}}{\partial X \partial Y}(X, Y) \\
\frac{\partial^{2} U_{\theta, \delta}}{\partial X \partial Y}(X, Y) & \frac{\partial^{2} U_{\theta, \delta}}{\partial Y^{2}}(X, Y)
\end{array}\right|>0 \\
\operatorname{tr} \operatorname{Hess} U_{\theta, \delta}(X, Y) & =\frac{\partial^{2} U_{\theta, \delta}}{\partial X^{2}}(X, Y)+\frac{\partial^{2} U_{\theta, \delta}}{\partial Y^{2}}(X, Y)>0 .
\end{aligned}
$$

Let us compute the partial second derivatives of $U_{\theta, \delta}$ :

$$
\begin{aligned}
\frac{\partial^{2} U_{\theta, \delta}}{\partial X^{2}}(X, Y) & =\int w(\xi, \eta) \frac{(Y+\eta)^{2}}{\left((X+\xi)^{2}+(Y+\eta)^{2}\right)^{3 / 2}} d \xi d \eta \\
\frac{\partial^{2} U_{\theta, \delta}}{\partial Y^{2}}(X, Y) & =\int w(\xi, \eta) \frac{(X+\xi)^{2}}{\left((X+\xi)^{2}+(Y+\eta)^{2}\right)^{3 / 2}} d \xi d \eta \\
\frac{\partial^{2} U_{\theta, \delta}}{\partial X \partial Y}(X, Y) & =\int-w(\xi, \eta) \frac{(X+\xi)(Y+\eta)}{\left((X+\xi)^{2}+(Y+\eta)^{2}\right)^{3 / 2}} d \xi d \eta
\end{aligned}
$$

From these expressions, we deduce easily that $\operatorname{tr} \operatorname{Hess} U_{\theta, \delta}(X, Y)>0$. Then using the identities $\left(\int f(\xi) d \xi\right)\left(\int g(\xi) d \xi\right)=\iint f(\xi) g(\eta) d \xi d \eta=\iint f(\eta) g(\xi) d \xi d \eta$ we have:

$$
\operatorname{det} \operatorname{Hess} U_{\theta, \delta}(X, Y)=\frac{\partial^{2} U_{\theta, \delta}}{\partial X^{2}}(X, Y) \frac{\partial^{2} U_{\theta, \delta}}{\partial Y^{2}}(X, Y)-\left(\frac{\partial^{2} U_{\theta, \delta}}{\partial X \partial Y}(X, Y)\right)^{2}
$$




$$
\begin{aligned}
& =\iint \frac{\left(Y+\eta_{1}\right)^{2}\left(X+\xi_{2}\right)^{2}-\left(X+\xi_{1}\right)\left(Y+\eta_{1}\right)\left(X+\xi_{2}\right)\left(Y+\eta_{2}\right)}{\left(\left(X+\xi_{1}\right)^{2}+\left(Y+\eta_{1}\right)^{2}\right)^{3 / 2}\left(\left(X+\xi_{2}\right)^{2}+\left(Y+\eta_{2}\right)^{2}\right)^{3 / 2}} d \xi_{1} d \eta_{1} d \xi_{2} d \eta_{2} \\
& =\frac{1}{2} \iint \frac{\left(\left(Y+\eta_{1}\right)\left(X+\xi_{1}\right)-\left(Y+\eta_{2}\right)\left(X+\xi_{1}\right)\right)^{2}}{\left(\left(X+\xi_{1}\right)^{2}+\left(Y+\eta_{1}\right)^{2}\right)^{3 / 2}\left(\left(X+\xi_{2}\right)^{2}+\left(Y+\eta_{2}\right)^{2}\right)^{3 / 2}} d \xi_{1} d \eta_{1} d \xi_{2} d \eta_{2} \\
& >0 .
\end{aligned}
$$

where $w\left(\xi_{1}, \eta_{1}\right) w\left(\xi_{2}, \eta_{2}\right)$ has been omitted to make the notation clearer.

\section{A.2 Moment Equilibria}

Having established the force equilibrium, we proceed to express it as a function of $\theta, \delta$. A.2.a Equilibrium Curve. We denote by $\left(X^{*}(\theta, \delta), Y^{*}(\theta, \delta)\right)$ the unique force equilibrium relative to $\theta$ and by $\left(x^{*}(\theta, \delta), y^{*}(\theta, \delta)\right)$ its expression in the $(x, y, \theta)$ system of coordinates:

$$
\begin{aligned}
& x^{*}(\theta, \delta)=\cos \theta X^{*}(\theta, \delta)-\sin \theta Y^{*}(\theta, \delta) \\
& y^{*}(\theta, \delta)=\sin \theta X^{*}(\theta, \delta)+\cos \theta Y^{*}(\theta, \delta) .
\end{aligned}
$$

We call equilibrium curve of parameter $\delta$ the curve $\left\{\left(x^{*}(\theta, \delta), y^{*}(\theta, \delta)\right), \theta \in \mathbf{S}^{1}\right\}$ of force equilibria.

When $\delta=0$ (pure radial field), due to the radial symmetry of the field, the set of equilibrium configurations is generated by the rotations of the part about one of its points called the pivot point [11].

Proposition $3 X^{*}, Y^{*}, x^{*}, y^{*}$ are continuously differentiable.

Proof: The proof of this proposition is based on the implicit function theorem. Let us define the following function from $\mathbb{R}^{4}$ into $\mathbb{R}^{2}$ :

$$
F:(X, Y) \rightarrow\left(\begin{array}{c}
\frac{\partial U}{\partial X}(X, Y, \theta, \delta) \\
\frac{\partial U}{\partial Y}(X, Y, \theta, \delta)
\end{array}\right)
$$

$\left(X^{*}, Y^{*}\right)$ minimizes the potential function $U_{\theta, \delta}$ for constant $\theta$ and $\delta$, and therefore fits the following implicit representation:

$$
F\left(X^{*}, Y^{*}, \theta, \delta\right)=0
$$

$F$ is continuously differentiable and the differential of the partial function $F_{\theta, \delta}$ of the variables $(X, Y)$ is exactly the Hessian of $U_{\theta, \delta}$. From Proposition 2, this differential is invertible everywhere. All the hypotheses of the implicit function theorem are thus satisfied, and 
therefore $X^{*}(\theta, \delta)$ and $Y^{*}(\theta, \delta)$ are continuously differentiable. From relations $(7)$ and $(8)$, $x^{*}$ and $y^{*}$ are also continuously differentiable.

Let us now denote by $U_{\delta}^{*}(\theta)$ the minimum value of the potential function for each $\theta$. Then it is straightforward that $(X, Y, \theta)$ is a local minimum of $U_{\delta}$ if and only if $\theta$ is a local minimum of $U_{\delta}^{*}$ and $X=X^{*}(\theta, \delta)$ and $Y=Y^{*}(\theta, \delta)$. The following proposition establishes a relation between the derivative of $U_{\delta}^{*}$ and the position in the plane of the force equilibrium.

Proposition 4 For any $\theta \in \mathbf{S}^{1}$,

$$
\frac{d U_{\delta}^{*}}{d \theta}(\theta)=\delta W x^{*}(\theta, \delta)
$$

Proof: In this proof we omit $\delta$ in the expressions of $X^{*}$ and $Y^{*}$ to make the notation simpler. By definition $U_{\delta}^{*}(\theta)=U_{\delta}\left(X^{*}(\theta), Y^{*}(\theta), \theta\right)$. Differentiating this expression wrt to $\theta$ leads to

$$
\begin{aligned}
\frac{d U_{\delta}^{*}}{d \theta}(\theta)= & \frac{\partial U_{\delta}}{\partial X}\left(X^{*}(\theta), Y^{*}(\theta), \theta\right) \frac{d X^{*}}{d \theta}(\theta)+\frac{\partial U_{\delta}}{\partial Y}\left(X^{*}(\theta), Y^{*}(\theta), \theta\right) \frac{d Y^{*}}{d \theta}(\theta)+ \\
& \frac{\partial U_{\delta}}{\partial \theta}\left(X^{*}(\theta), Y^{*}(\theta), \theta\right) \\
= & \frac{\partial U_{\delta}}{\partial \theta}\left(X^{*}(\theta), Y^{*}(\theta), \theta\right) \\
= & \delta W\left(\cos \theta X^{*}(\theta)-\sin \theta Y^{*}(\theta)\right) \\
= & \delta W x^{*}(\theta, \delta),
\end{aligned}
$$

since the partial derivatives of $U_{\delta}$ wrt $X$ and $Y$ are null at $\left(X^{*}, Y^{*}\right)$.

Proposition 4 states that a stable equilibrium configuration corresponds to a value of $\theta$ where the equilibrium curve crosses the $\mathrm{y}$-axis from $x<0$ to $x>0$. Figure 10 (bottom left) shows the value of $U_{\delta}^{*}$ along the equilibrium curve for the ratchet part in the same figure and illustrates perfectly the linearity of the relation between $\frac{d U_{\delta}^{*}}{d \theta}$ and $x^{*}$. Indeed, it can be easily checked that the torque $\mathbf{M}$ is equal to the partial derivative of $U_{\delta}$ w.r.t. $\theta$.

A.2.b Unique Global Equilibrium. We combine our results in propositions 2, 3, and 4 to establish the concluding theorem of this section.

Theorem 5 For any compact part $w$, if $\left(X^{*}(\theta, 0), Y^{*}(\theta, 0)\right) \neq(0,0)$ (i.e. the center of mass and the pivot point are distinct) then there exists $\delta>0$ such that $w$ has a unique stable equilibrium configuration under the potential field $U_{\delta}$. 
Proof: First, let us notice that the curve $\left(X^{*}(\theta, 0), Y^{*}(\theta, 0)\right)$ is reduced to a point since when $\delta=0$, the potential field $U_{\delta}$ does not depend on $\theta$. Let us express this point in polar coordinates:

$$
\begin{aligned}
& X^{*}(\theta, 0)=R \cos \varphi \\
& Y^{*}(\theta, 0)=R \sin \varphi .
\end{aligned}
$$

Then if $\left(X^{*}, Y^{*}\right) \neq(0,0)$, from relations $(7)$ and $(8)$, the curve $\left(x^{*}(\theta, 0), y^{*}(\theta, 0)\right)$ is a circle centered on $(0,0)$ (Figure 7$)$. We have:

$$
\begin{aligned}
& x^{*}(\theta, 0)=R \cos (\theta+\varphi) \\
& y^{*}(\theta, 0)=R \sin (\theta+\varphi) .
\end{aligned}
$$

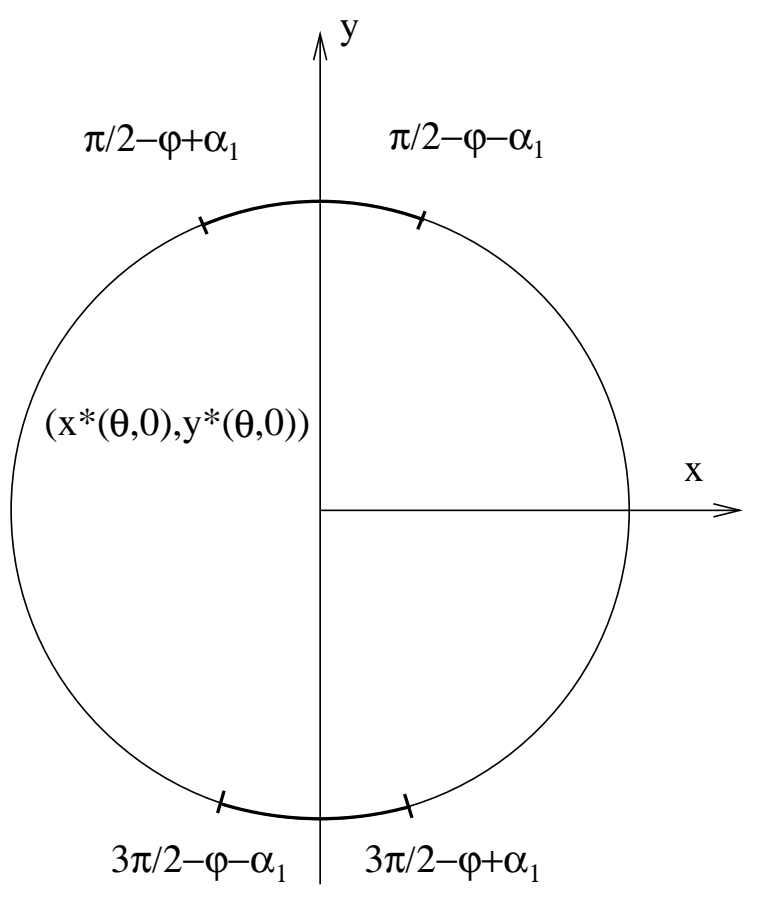

Fig. 7. Decomposition of the equilibrium curve for $\delta=0$ into four intervals.

The current proof is based on the continuity of the functions $x^{*}$ and $y^{*}$ and their derivatives. We proceed in two steps: near $\pi / 2-\varphi$ and $3 \pi / 2-\varphi$, where $x^{*}(\theta, 0)$ crosses 0 , the variation of the tangent vector to the curve $\left(x^{*}(\theta, \delta), y^{*}(\theta, \delta)\right)$ can be made sufficiently small in order to prevent the curve to cross twice the y-axis. For the remaining values of $\theta$, the variation of the position of the curve can be bounded in such a way that the curve cannot cross the y-axis. The complete proof follows. 
Let us recall that $\partial x^{*} / \partial \theta(\theta, \delta)$ is a continuous function and that $\partial x^{*} / \partial \theta(-\varphi+\pi / 2,0)=$ $-R$ and $\partial x^{*} / \partial \theta(3 \pi / 2-\varphi, 0)=R$. Therefore there exists $\alpha_{1}>0$ and $\delta_{1}>0$ such that

$$
\begin{array}{ll}
\forall \delta<\delta_{1}, \forall \theta \in\left[-\varphi+\pi / 2-\alpha_{1},-\varphi+\pi / 2+\alpha_{1}\right], & \frac{\partial x^{*}}{\partial \theta}(\theta, \delta)<0 \\
\forall \delta<\delta_{1}, \forall \theta \in\left[-\varphi+3 \pi / 2-\alpha_{1},-\varphi+3 \pi / 2+\alpha_{1}\right], & \frac{\partial x^{*}}{\partial \theta}(\theta, \delta)>0 .
\end{array}
$$

These inequalities imply that the equilibrium curve does not cross more than once the $\mathrm{y}$-axis on the corresponding intervals of $\theta$.

We are going now to show that for the remaining values of $\theta$, there exists a $\delta$ small enough such that the corresponding part of the equilibrium curve does not cross the y-axis. To make the notation clearer, let us define the following compact set

$$
I=\left[-\varphi+\pi / 2+\alpha_{1},-\varphi+3 \pi / 2-\alpha_{1}\right] \cup\left[-\varphi+3 \pi / 2+\alpha_{1},-\varphi+5 \pi / 2-\alpha_{1}\right] .
$$

Then for $\delta=0$ and $\theta \in I$, the equilibrium curve stays at a strictly positive distance from the y-axis:

$$
\alpha_{2}=\operatorname{Inf}\left\{\left|x^{*}(\theta, 0)\right|, \theta \in I\right\}>0
$$

$x^{*}$ is continuous, thus its restriction to the compact set $I \times\left[0, \delta_{1}\right]$ is uniformly continuous. Therefore, there exists a constant $\delta_{2}>0$ such that

$$
\forall \theta \in I, \forall \delta \in\left[0, \delta_{2}\right],\left|x^{*}(\theta, \delta)-x^{*}(\theta, 0)\right|<\alpha_{2}
$$

and this condition ensures that the equilibrium curves does not cross the y-axis for $\theta \in I$ and $\delta<\delta_{2}$.

Therefore, for any $\delta<\min \left(\delta_{1}, \delta_{2}\right)$, the equilibrium curve crosses the y-axis exactly twice. Once in each direction.

\section{B. Prediction of Equilibria}

The previous computation shows that if a part has a pivot point different from the center of mass, then there exists a small value of $\delta$ to uniquely orient this part. However, this does not mean that there exists one unique value of $\delta$ orienting any part. In other words, the combination of a radial unit field and a gravitational field is a strategy that can orient almost any part, but for each part the maximum $\delta$ is different.

Figure 8 shows equilibrium curves for the ratchet for different values of $\delta$. In this example, we can see that for large $\delta$, the equilibrium curve crosses the y-axis several times, and thus the minimum is not unique anymore. An annealing process may be used to determine $\delta$. The process starts with a value of $\delta$ just below 1 . This causes the part 
to be centered and oriented quickly. By reducing $\delta$ we ensure that eventually we obtain a field that uniquely orients the part.

Alternatively, we can determine the maximum value for $\delta$ for which the equilibrium is unique by performing a binary search. By using numerical methods, we observe that for the ratchet for all $\delta$ values up to 0.46 the equilibrium is unique. This is demonstrated in Figure 8. Numerous simulation runs were performed to observe the behavior of the ratchet in the field $\mathbf{r}+0.46 \mathrm{~g}$. It consistently reaches the unique final position. Some of these simulation runs are shown in Figure 9.

Figure 10 combines all these observations for a field $\mathbf{r}+0.3 \mathrm{~g}$.

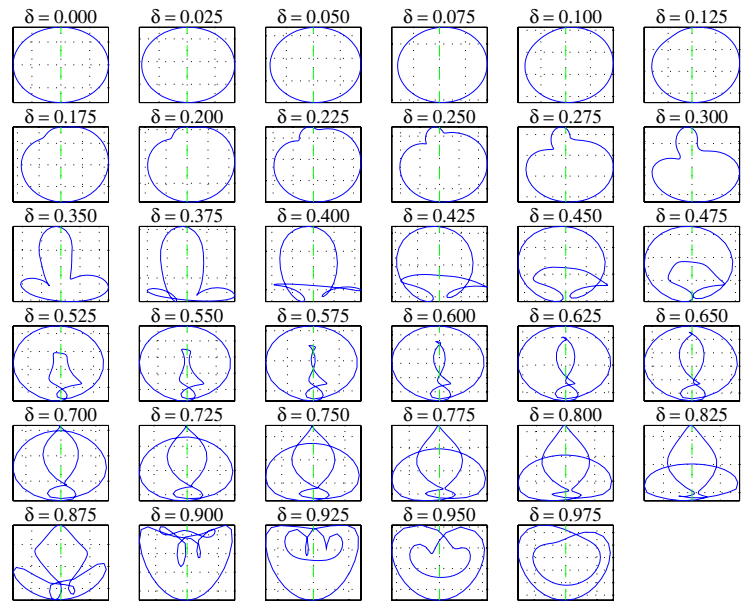

(a)

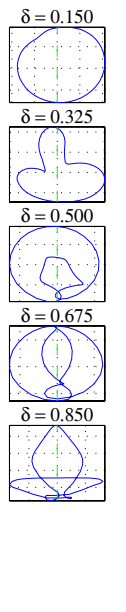

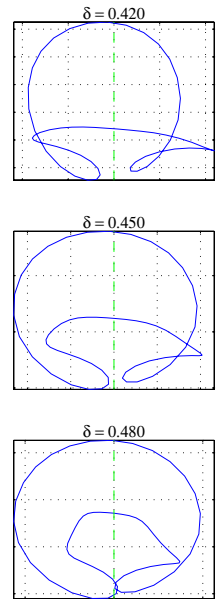
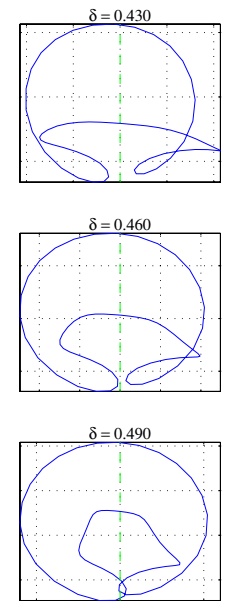

(b)
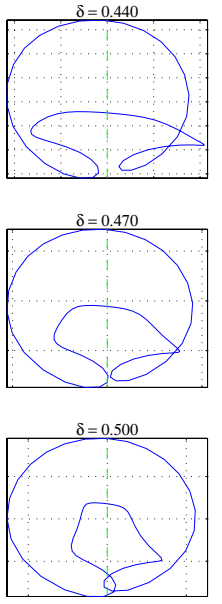

Fig. 8. Detailed equilibrium curves for the ratchet: (a) Curves from $\delta=0$ to $\delta=0.975$, increment 0.025 .

(b) Curves from $\delta=0.42$ to $\delta=0.50$, increment 0.01 . We observe that up to $\delta=0.46$ the curve has only two intersections with the $y$-axis, hence the equilibrium is unique.

\section{IMPLEMENTATION}

The previous sections show that there exist universal feeder/orienter devices that can uniquely position almost any part. We now briefly investigate practical issues on building such devices. To this end we pose two key questions:

- How difficult is it to build devices that implement programmable force fields?

- How efficient is a universal feeder/orienter device in practice?

The first question concerns the initial setup cost as compared, e.g., with a vibratory bowl feeder or a robotic parts feeder. The second question addresses the issue that even though unique equilibria exist for almost all parts, it is not obvious a priori how quickly these equilibria will be reached. To obtain an answer to these questions we have built a 

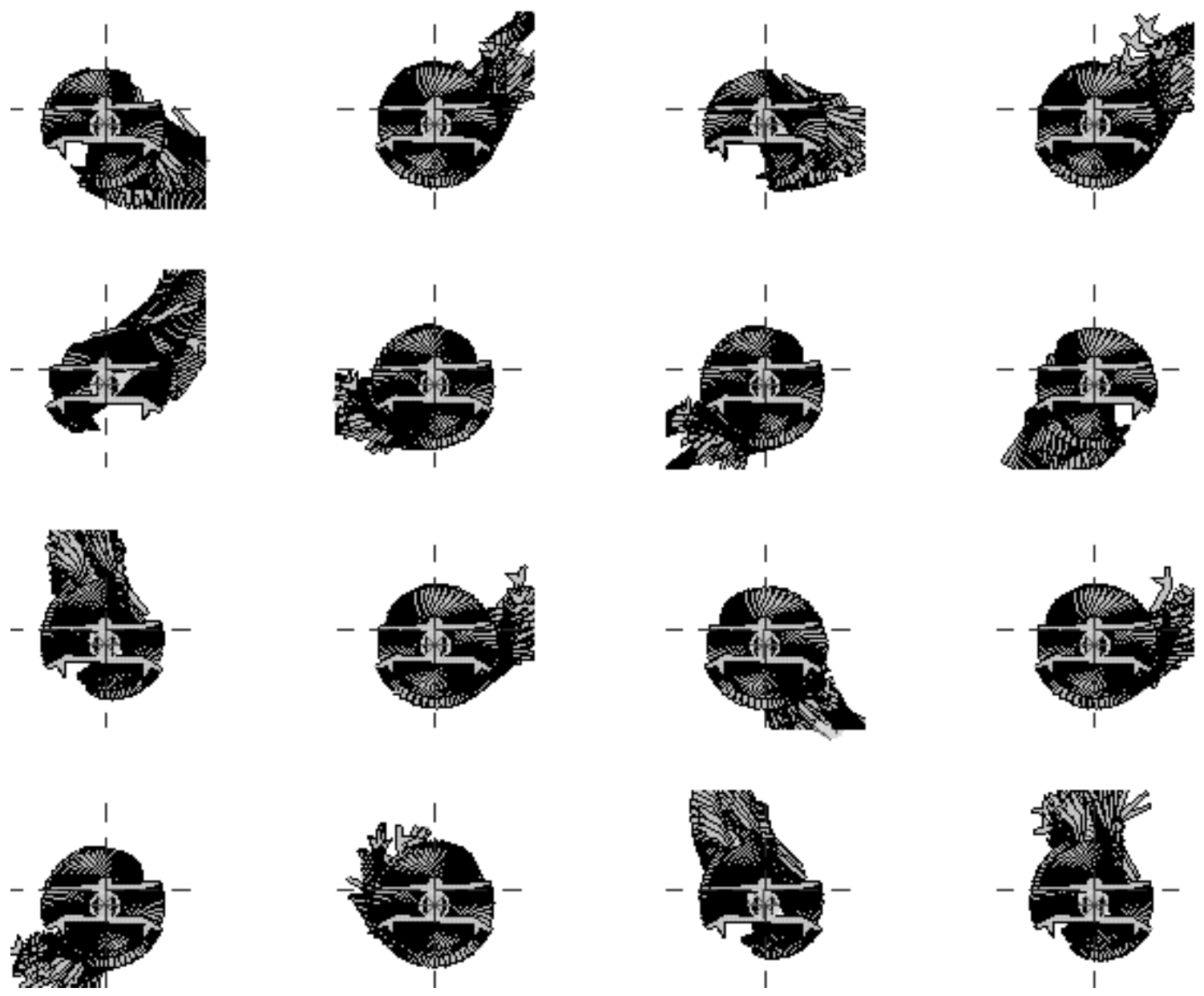

Fig. 9. Simulation runs for the ratchet in the field $\mathbf{r}+0.46 \mathrm{~g}$. In all runs the part reaches the same final pose.

comprehensive simulation and analysis system, and we have investigated multiple designs that implement prototype devices for programmable force fields.

\section{A. Simulation}

We have implemented a sophisticated simulator for programmable force vector fields in MATLAB. The system is capable of exact calculation of the force acting on polygonal parts in various fields, including squeeze, unit radial, gravity fields, and combinations thereof. To calculate the force acting on a polygon in the field, the polygon is triangulated and the force field is integrated over the individual areas. This can be done without numerical integration since there exist closed-form integrals for all these fields. To predict the part motion in the field, we have implemented a full dynamic simulator that includes inertia, viscous damping, and Coulomb friction. Force equilibria are determined numerically by 

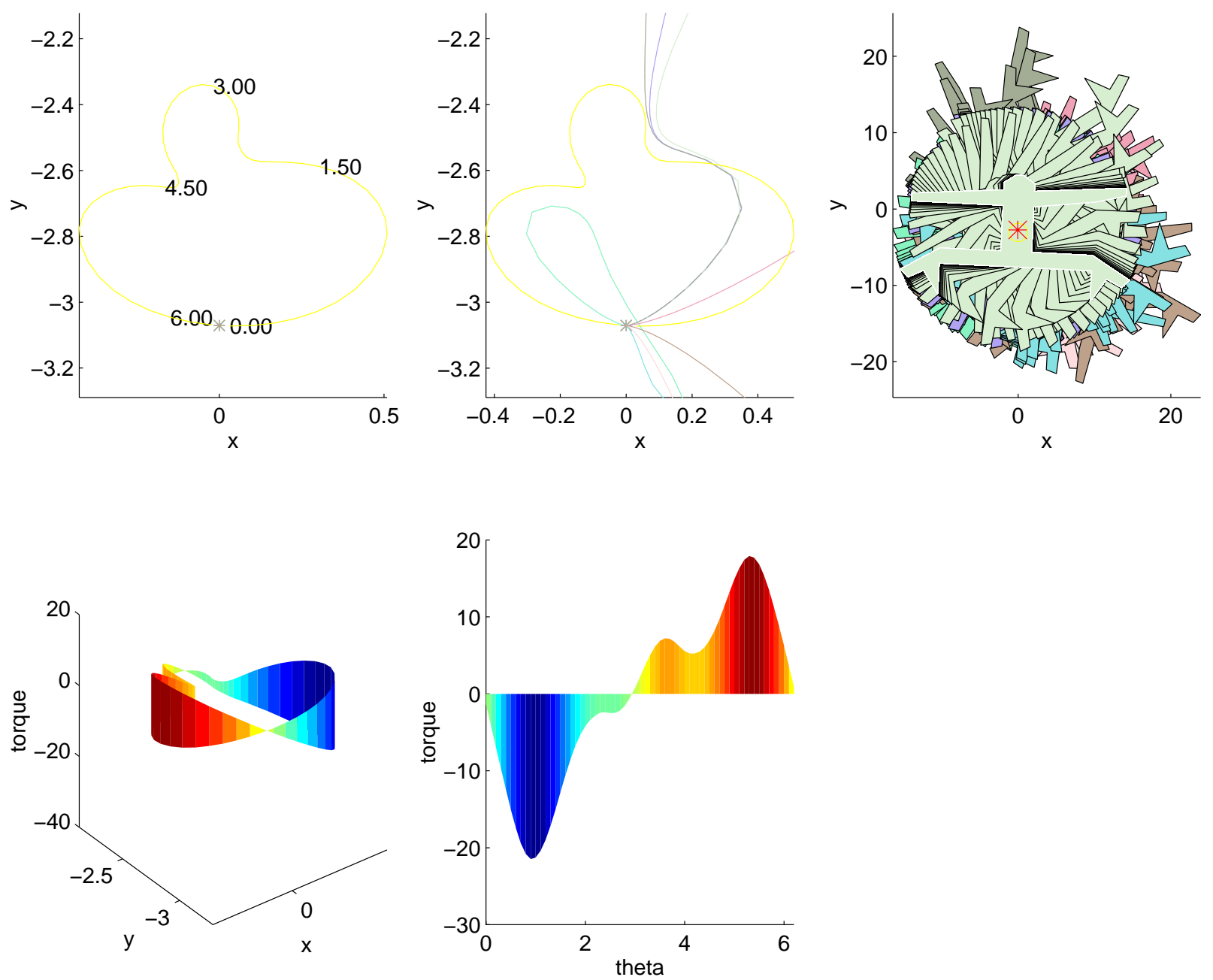

Fig. 10. Analysis of the radial-gravity field $F_{\delta}$ with the ratchet part. From left to right, top to bottom: - Equilibrium curve for $\delta=0.3$. Each point on this curve corresponds to a specific $\theta$ value.

- Equilibrium curve with simulated trajectories of the ratchet. The center of mass always reaches the unique stable equilibrium (corresponding to the lower intersection of the curve with the $x$-axis).

- Multiple simulation runs. The ratchet always reaches the same stable total equilibrium.

- Equilibrium curve with corresponding torques.

- Torque on $E_{P}$ as a function of $\theta$. 
solving the constraints $\mathbf{F}=0$ as given in equation 1. Pivot points are also determined numerically.

Figures 9 and 10 consist of output from the software package and include dynamic simulation, numerical computation of force equilibria, and computation of torque when the part already is in force equilibrium (i.e., the torque associated with each point on the equilibrium curve). For the torque calculation see the last part of Figure 10.

\section{B. Device Construction}

In Sections I-A and I-B we have already mentioned some device designs that implement programmable force fields. The idea of open-loop parts feeding is particularly attractive when dealing with very small or microfabricated parts, where precise feedback is difficult or extremely expensive. It also opens the opportunity for massively parallel positioning and assembly: since no control is required, the positioning process can be parallelized without communication overhead.

Towards this end, various researchers have demonstrated microfabricated actuator arrays based on MEMS (micro electro mechanical system) technology. These devices consist of a surface with potentially thousands or even millions of microscopic actuators, each of them capable of generating a unit force in a specific direction $[40,3,27,14,33$, for example].

While MEMS actuator arrays may be useful to implement force fields that require high spatial resolution, alternative (macroscopic) designs are possible as well. In the following subsections we give some specific design ideas.

\section{B.1 Elliptic Fields}

The realization of elliptic fields could be achieved with MEMS actuator arrays [10, 13], or arrays of motors [35], and possibly with vibrating plates [6]. The main challenge for vibrating plates will be to obtain a surface that approximates the elliptic force profile with sufficient spatial resolution. Microscopic (MEMS) or macroscopic (motor) actuator arrays offer alternatives. Note that individual control of the actuators is not necessary; control by rows and columns only is sufficient. Furthermore, the proposed force field could be implemented with a technology that allows the specification of a force only in one of the $x$ or $y$ directions at each pixel/actuator. Then two arrays, one controlled only in the $x$ direction and the other controlled only in the $y$ direction, can be "interleaved." If the arrays are dense, the resulting force will be a force with the desired magnitude and direction. The main challenge for micro actuators remains the generation and control of forces over a sufficiently large range of force magnitudes. 


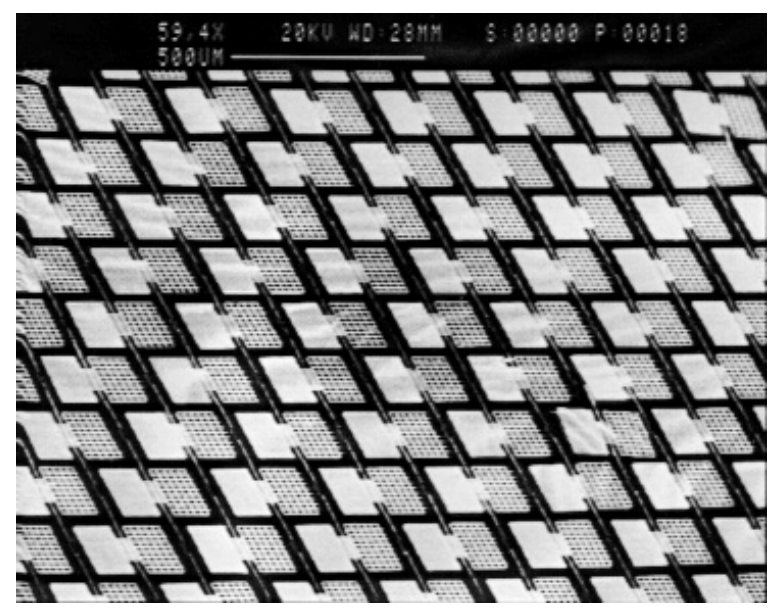

Fig. 11. Unidirectional MEMS actuator array build on a silicon wafer. Each actuator is about $0.2 \mathrm{~mm}$ in size.

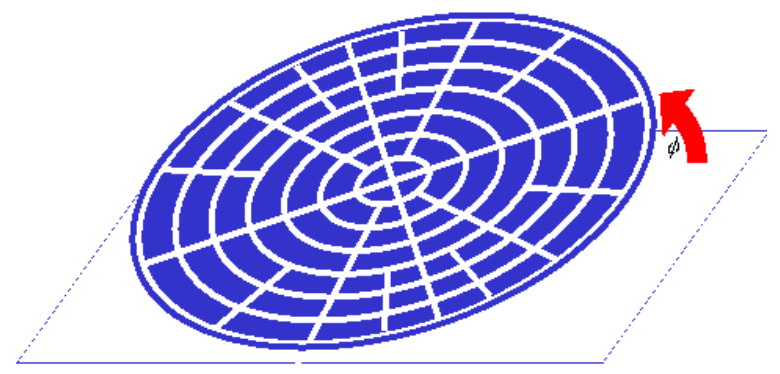

Fig. 12. Conceptual design of an actuator array that implements a combined radial-gravity field. Individual actuators are tiled in a circular array pattern. The array is tilted between $\phi=0^{\circ}$ and $45^{\circ}$ to add a gravity component $\delta \mathrm{g}$. Under some simplifying assumptions $\delta=\tan \phi$.

\section{B.2 Universal Fields}

A prototype unidirectional array was built by Böhringer et al. [10] (see Figure 11). This array can generate a unit gravity field. Its design could be modified such that the actuators are arranged in a circular pattern, which would result in a unit radial field. The variable gravity field could then be added simply by tilting the array accordingly (see Figure 12). Hence such a device would be relatively easy to build. The key observation is that with current MEMS technology it is easy to build actuator arrays with high spatial resolution ( $\ll 1 \mathrm{~mm}$ ) and constant force, but it is difficult to build actuators with variable force. In addition, MEMS actuators can be easily arranged into arbitrary patterns (in particular, a radial pattern). Hence it is easy to build arrays that implement unit radial fields. 
Alternatively, a resonating speaker, or a vibrating disk-shaped plate that is fixed at the center, might be used to create a radial force field.

\section{DISCUSSION}

This paper proves the existence of devices for parts positioning and orienting that can bring arbitrary (non-symmetric) parts into exactly one or two stable equilibria. These devices are extremely simple: they do not require a feedback control, a clock, synchronization, or programming. Their functioning principle is based on force vector fields. Such a device could revolutionize industrial and precision parts handling.

This result opens the door for a multitude of new questions, some of which are briefly outlined below.

\section{Open Questions}

.2.a Parallelism. So far we have considered only the equilibria of one part in a force field. But what happens if two parts are placed into the field simultaneously? It is conceivable that the parts will settle in predictable configurations. This effect could be exploited for automated assembly.

When parts are initially placed far enough apart, it may be possible to implement several radial-gravity fields next to each other to achieve parallel positioning. This issue is particularly interesting since there is no overhead for parallelism in such a device, as no communication and control is required.

2.b Symmetric Parts. In Section IV we have shown that elliptic fields achieve two equilibria for any part with $s_{11} \neq 0$ and $s_{20} \neq s_{02}$. Parts that do not satisfy this condition will be in neutral orientation equilibrium once their centers of mass reach the center of the elliptic field. Since the above conditions are not met for parts with rotational symmetry, these parts cannot be uniquely oriented in an elliptic field.

Similarly, Theorem 5 requires that the pivot point and center of mass of a part do not coincide. Thus, this result does not apply to rotationally symmetric parts such as, e.g., squares or hexagons. However, simulation results indicate that symmetric parts may still reach a unique equilibrium up to part symmetry. In case a part is symmetric, the user may not care about multiple equilibria as long as there exists no noticeable difference in the final poses. Therefore we generalize Theorem 5 to obtain the following conjecture: $A$ radial-gravity field uniquely poses any part up to part symmetry.

2.c Large $\delta$ Values. We have shown that there always exists a $\delta_{\max }$ such that for all $0<\delta<\delta_{\max }$ we obtain a unique equilibrium. Figure 8 shows that for $\delta>\delta_{\max }$ 
the equilibrium curve becomes more complicated, causing multiple equilibria. However, as $\delta$ approaches 1 the curve becomes simpler again. Since higher $\delta$ values imply faster convergence, it would be interesting to know whether unique equilibria can be found for $\delta$ close to 1 .

\section{ACKNOWLEDGMENTS}

Work on this paper by Karl Böhringer and Bruce Randall Donald has been supported in part by the National Science Foundation under Grant Nos. IRI-8802390, IRI-9000532, IRI9201699, IRI-9530785, IRI-9896020, NSF EIA-9901407, NSF IIS-9906790, NSF 9802068, NSF CDA-9726389, NSF EIA-9818299, NSF CISE/CDA-9805548, by an equipment grant from Microsoft Research, by a Presidential Young Investigator award to Bruce Donald, by an NSF/ARPA Small Grant for Exploratory Research No. IRI-9403903, by an NSF CISE Postdoctoral Associateship No. CDA-9705022 and an NSF CAREER Award No. ECS9875367 to Karl Böhringer, and in part by the Air Force Office of Sponsored Research, the Mathematical Sciences Institute, Intel Corporation, and AT\&T Bell laboratories. Work on this paper by Lydia Kavraki and Florent Lamiraux has been supported in part by NSF IRI-970228 and NSF CISE SA1728-21122N. The authors would like to thank Eric Babson, Mike Erdmann, Leo Guibas, Jean-Claude Latombe, and Andy Ruina for valuable discussions.

\section{ReFerences}

[1] T. L. Abell and M. Erdmann. A universal parts feeder, 1996. Personal communication / in preparation.

[2] S. Akella, W. H. Huang, K. M. Lynch, and M. T. Mason. Planar manipulation on a conveyor by a one joint robot with and without sensing. In International Symposium of Robotics Research (ISRR), 1995.

[3] M. Ataka, A. Omodaka, and H. Fujita. A biomimetic micro motion system. In Transducers - Digest Int. Conf. on Solid-State Sensors and Actuators, pages 38-41, Pacifico, Yokohama, Japan, June 1993.

[4] D. Biegelsen, W. Jackson, A. Berlin, and P. Cheung. Air jet arrays for precision positional control of flexible media. In Int. Conf. on Micromechatronics for Information And Precision Equipment (MIPE'97), Tokyo, Japan, July 1997.

[5] K.-F. Böhringer, V. Bhatt, B. R. Donald, and K. Y. Goldberg. Algorithms for sensorless manipulation using a vibrating surface. Algorithmica, 26(3-4), March/April 2000. Special Issue on Algorithmic Foundations of Robotics. Accepted for publication. Forthcoming.

[6] K.-F. Böhringer, V. Bhatt, and K. Y. Goldberg. Sensorless manipulation using transverse vibrations of a plate. In Proc. IEEE Int. Conf. on Robotics and Automation (ICRA), pages 1989-1996, Nagoya, Japan, May 1995.

[7] K.-F. Böhringer, R. G. Brown, B. R. Donald, J. S. Jennings, and D. Rus. Distributed robotic manipulation: Experiments in minimalism. In O. Khatib et al., editor, Experimental Robotics IV, Lecture Notes in Control and Information Sciences 223, pages 11-25. Springer Verlag, Berlin, 1997.

[8] K.-F. Böhringer, B. R. Donald, and D. Halperin. On the area bisectors of a polygon. Discrete and Computational Geometry, 22:269-285, 1999.

[9] K.-F. Böhringer, B. R. Donald, L. Kavraki, and F. Lamiraux. A single universal force field can uniquely orient non-symmetric parts. In 9th International Symposium on Robotics Research, pages 313-320, 1999. 
[10] K.-F. Böhringer, B. R. Donald, and N. C. MacDonald. Single-crystal silicon actuator arrays for micro manipulation tasks. In Proc. IEEE Workshop on Micro Electro Mechanical Systems (MEMS), pages 7-12, San Diego, CA, Feb. 1996.

[11] K.-F. Böhringer, B. R. Donald, and N. C. MacDonald. Upper and lower bounds for programmable vector fields with applications to MEMS and vibratory plate parts feeders. In International Workshop on Algorithmic Foundations of Robotics (WAFR), Toulouse, France, July 1996. Appeared in Algorithms for Robotic Motion and Manipulation, Jean-Paul Laumond and Mark Overmars (Eds), pages 255-276, A. K. Peters, Ltd, 1997.

[12] K.-F. Böhringer, B. R. Donald, and N. C. MacDonald. Programmable vector fields for distributed manipulation, with applications to MEMS actuator arrays and vibratory parts feeders. Int. Journal of Robotics Research, Feb. 1999.

[13] K.-F. Böhringer, B. R. Donald, N. C. MacDonald, G. T. A. Kovacs, and J. W. Suh. Computational methods for design and control of MEMS micromanipulator arrays. IEEE Computer Science and Engineering, pages 17-29, January-March 1997.

[14] K.-F. Böhringer, B. R. Donald, R. Mihailovich, and N. C. MacDonald. Sensorless manipulation using massively parallel microfabricated actuator arrays. In Proc. IEEE Int. Conf. on Robotics and Automation (ICRA), pages 826-833, San Diego, CA, May 1994.

[15] K.-F. Böhringer, K. Goldberg, M. B. Cohn, R. Howe, and A. Pisano. Parallel microassembly with electrostatic force fields. In Proc. IEEE Int. Conf. on Robotics and Automation (ICRA), Leuven, Belgium, May 1998.

[16] R. Brooks. A layered intelligent control system for a mobile robot. IEEE Journal of Robotics and Automation, RA(2), 1986.

[17] J. Canny and K. Goldberg. "RISC" for industrial robotics: Recent results and open problems. In Proc. IEEE Int. Conf. on Robotics and Automation (ICRA). IEEE, May 1994.

[18] P. Cheung, A. Berlin, D. Biegelsen, and W. Jackson. Batch fabrication of pneumatic valve arrays by combining mems with printed circuit board technology. In Proc. Symposium on Micro-Mechanical Systems, ASME International Mechanical Engineering Congress and Exhibition, pages 16-21, Dallas, TX, Nov. 1997.

[19] G. Chirikjian. Kinematic synthesis of mechanisms and robotic manipulators with binary actuators. $A S M E$ Journal of Mechanical Design, 117:573-580, December 1995.

[20] M. Coutinho and P. Will. The intelligent motion surface: a hardware/software tool for the assembly of mesoscale devices. In Proc. IEEE Int. Conf. on Robotics and Automation (ICRA), Albuquerque, New Mexico, Apr. 1997.

[21] M. Coutinho and P. Will. Using dynamic vector force fields to manipulate parts on an intelligent motion surface. In IEEE International Symposium on Automation and Task Planning, Los Angeles, CA, 1997.

[22] M. Coutinho and P. Will. A general theory for positioning and orienting 2d polygonal or curved parts using intelligent motion surfaces. In Proc. IEEE Int. Conf. on Robotics and Automation (ICRA), Leuven, Belgium, May 1998.

[23] B. R. Donald, J. Jennings, and D. Rus. Information invariants for distributed manipulation. In K. Goldberg, D. Halperin, J.-C. Latombe, and R. Wilson, editors, Algorithmic Foundations of Robotics (WAFR), pages 431-459. A. K. Peters, Ltd, Wellesley, MA, 1995.

[24] M. Erdmann. An exploration of nonprehensile two-palm manipulation: Planning and execution. In G. Giralt and G. Hirzinger, editors, Robotics Research, pages 16-27. Springer Verlag, 1996.

[25] M. A. Erdmann and M. T. Mason. An exploration of sensorless manipulation. IEEE Journal of Robotics and Automation, 4(4), Aug. 1988.

[26] M. A. Erdmann and M. T. Mason. Nonprehensile manipulation. In Robotic Motion and Manipulation, Toulouse, France, July 1996.

[27] H. Fujita. Group work of microactuators. In International Advanced Robot Program Workshop on Micromachine Technologies and Systems, pages 24-31, Tokyo, Japan, Oct. 1993.

[28] K. Y. Goldberg. Orienting polygonal parts without sensing. Algorithmica, 10(2/3/4):201-225, August/September/October 1993.

[29] B. Joffe. Manipulation and identification of objects by magnetic forces. In Proc. International Symposium 
on Magnetic Suspension Technology, NASA Conference Publication 3152 part 2, pages 617-639, Langley, VA, Aug. 1991.

[30] L. Kavraki. Part orientation with programmable vector fields: Two stable equilibria for most parts. In Proc. IEEE Int. Conf. on Robotics and Automation (ICRA), pages 20-25, Albuquerque, New Mexico, Apr. 1997.

[31] O. Khatib. Real time obstacle avoidance for manipulators and mobile robots. Int. Journal of Robotics Research, 5(1):90-99, Spring 1986.

[32] D. E. Koditschek and E. Rimon. Robot navigation functions on manifolds with boundary. Advances in Applied Mathematics, 1988.

[33] W. Liu and P. Will. Parts manipulation on an intelligent motion surface. In IEEE/RSJ Int. Workshop on Intelligent Robots \& Systems (IROS), Pittsburgh, PA, 1995.

[34] J. E. Luntz and W. Messner. A distributed control system for flexible materials handling. IEEE Control Systems, 17(1), Feb. 1997.

[35] J. E. Luntz, W. Messner, and H. Choset. Parcel manipulation and dynamics with a distributed actuator array: The virtual vehicle. In Proc. IEEE Int. Conf. on Robotics and Automation (ICRA), pages 1541-1546, Albuquerque, New Mexico, Apr. 1997.

[36] K. Lynch. Nonprehensile Robotic Manipulation. PhD thesis, The Robotics Institute, CMU, Pittsburgh, PA, 1996.

[37] T. McGeer. Passive dynamic walking. Int. Journal of Robotics Research, 1990.

[38] J. L. Meriam and L. G. Kraige. Engineering Mechanics - Statics. John Wiley and Sons, 4 edition, 1997.

[39] D. Miller. A rwelve-step program to more efficient robotitcs. AI Magazine, pages 60-63, 1993.

[40] K. S. J. Pister, R. Fearing, and R. Howe. A planar air levitated electrostatic actuator system. In Proc. IEEE Workshop on Micro Electro Mechanical Systems (MEMS), pages 67-71, Napa Valley, California, Feb. 1990.

[41] M. H. Raibert, J. K. Hodgins, R. R. Playter, and R. P. Ringrose. Animation of legged maneuvers: jumps, somersaults, and gait transitions. Journal of the Robotics Society of Japan, 11(3):333-341, 1993.

[42] J. Reif and H. Wang. Social potential fields: A distributed behavioral control for autonoomous robots. In K. Goldberg, D. Halperin, J.-C. Latombe, and R. Wilson, editors, Algorithmic Foundations of Robotics (WAFR), pages 431-459. A. K. Peters, Ltd, Wellesley, MA, 1995.

[43] D. Reznik, S. Brown, and J. F. Canny. Dynamic simulation as a design tool for a microactuator array. In Proc. IEEE Int. Conf. on Robotics and Automation (ICRA), Albuquerque, NM, Apr. 1997.

[44] D. Reznik and J. F. Canny. Universal part manipulation in the plane with a single horizontally vibrating plate. In P. K. Agarwal, L. Kavraki, and M. Mason, editors, Robotics: The Algorithmic Perspective. A. K. Peters, Ltd, Wellesley, MA, 1998.

[45] D. Reznik, J. F. Canny, and K. Y. Goldberg. Analysis of part motion on a longitudinally vibrating plate. In IEEE/RSJ Int. Workshop on Intelligent Robots \&\& Systems (IROS), Grenoble, France, Sept. 1997.

[46] E. Rimon and D. Koditschek. Exact robot navigation using artificial potential functions. IEEE Transactions on Robotics and Automation, 8(5), October 1992.

[47] B. Roth, J. Rastegar, and V. Scheinman. On the design of computer controlled manipulators. CISM-IFTMM Symp. on Theory and Practice of Robots and Manipulators, pages 93-113, 1973.

[48] B.-Z. Sandler. Robotics: Designing the Mechanisms for Automated Machinery. Prentice Hall, 1991.

[49] J. W. Suh, R. B. Darling, K. F. Böhringer, H. Baltes, B. R. Donald, and G. T. A. Kovacs. CMOS integrated organic ciliary array for general-purpose micromanipulation tool for small objects. Journal of Microelectromechanical Systems, 1998. Submitted for review.

[50] J. Wiegley, K. Goldberg, M. Peshkin, and M. Brokowski. A complete algorithm for designing passive fences to orient parts. In Proc. IEEE Int. Conf. on Robotics and Automation (ICRA), pages 1133-1139, Minneapolis, MN, Apr. 1996. 